Erschienen in: Born, Joachim/Stickel, Gerhard (Hrsg.): Deutsch als Verkehrssprache in Europa. - Berlin, New York: de Gruyter, 1993. S. 262-272. (Institut für deutsche Sprache. Jahrbuch 1992)

\title{
HUBERT EICHHEIM
}

\section{Deutsch in einem zusammenwachsenden Europa - aus der Sicht des Goethe-Instituts}

You don't have to learn a foreign language, all you have to do is to shout a little louder in English - Das Zitat stammt aus einer Anzeige des britischen Department of Trade and Industry und könnte auf das Deutsche übertragen von dem einen oder anderen deutschen Politiker stammen. Da es aber aus England kommt, wo Witz, lronie und tiefere Bedeutung nach unserer Schulbuchvorstellung zu Hause sind, lohnt es sich, dem Satz auf den Grund zu gehen. Er läßt nämlich drei unterschiedliche Deutungen zu, eine ethnozentrische, eine ethnopathische und eine eurozentrierte. Tatsächlich finden wir alle drei Haltungen in jedem europäischen Land jeweils in unterschiedlichem Mischverhältnis.

Die ethnozentrische repräsentiert die vierschrötige, unsensible Haltung, die sich etwa so ausdrückt: Wozu sollen wir uns auf die anderen Europäer einstellen, wo wir doch das zivilisatorisch am weitesten entwickelte Land sind, über die mächtigste lndustrie verfügen, die schnellsten Autos produzieren, die pünktlichsten Eisenbahnen haben, die eleganteste Sprache sprechen, auf die höchst entwickelten kulturellen Leistungen zurückblicken, über das leistungsfähigste Sozialsystem verfügen, und was immer jedes einzelne Land zu seinen Gunsten vorbringen kann. Diese Haltung findet sich hauptsächlich in den großen Ländern, deren Sprachgrenzen über die Landesgrenzen hinausgehen, also in Großbritannien, in Deutschland und in Frankreich, wo man zwar Fremdsprachen in der Schule lernt, aber oft so, daB man garantiert nichts damit anfangen kann, zumal es auch nicht als schicklich gilt, einem Ausländer in einer anderen als der eigenen Sprache auf der Straße Auskunft zu erteilen.

Die ethnopathische Haltung drückt aus, daß man sich gewisser Defizite durchaus bewuBt ist und sie ausgleicht, indem man entweder unkritisch alles übernimmt, von dem man glaubt, daß es zum Fortschritt gehört, oder aus angeschlagenem Nationalstolz sich in chauvinistischer Kraftmeierei ergeht. Diese Einstellung findet sich vor allem in Südeuropa, etwa in Griechenland, wo man schon wegen des Tourismus auf die Beherrschung von Fremdsprachen angewiesen wäre, aber in den öffentlichen Schulen nur eine Fremdsprache zuläßt zugunsten einer nationalen Nabelschau mit Griechisch (neu und alt), Aufsatz, Geschichte und Religion, die mehr als 2/3 des gesamten Stundenplans in Beschlag nehmen.

Die eurozentrierte Haltung schließlich ist das, was in den offiziellen Willenserklärungen zu Europa steht und sicher nicht in jedem 
Fall und nur als Lippenbekenntnis zu werten ist. Sie ist vor allen in den kleineren nordeuropäischen Ländern anzutreffen, aber auch in den Ländern Mittel- und Osteuropas. Wie immer man die zitierte Anzeige interpretieren mag, drückt sie doch eine gewaltige Unruhe aus, die wohl alle Nationen in Ost- und Westeuropa erfaft hat, die am gemeinsamen Binnenmarkt früher oder später teilnehmen wollen und denen die Richtigkeit des Satzes „La meilleure langue commerçiale est celle du client" einleuchtet (Marc Blancpain, ehemaliger Direktor der Alliance Française). Diese Unruhe hat ein gewaltiges Potential an Überlegungen und Aktivitäten in allen europäischen Ländern auf allen gesellschaftlichen und politischen Ebenen vitalisiert, die zu einer Neuordnung des Fremdsprachenunterrichts in seiner ganzen Dimension führen dürften.

Das Goethe-Institut mit seinem Auftrag zur „Pflege der deutschen Sprache im Ausland und zur Förderung der internationalen kulturellen $\mathrm{Zu}$ sammenarbeit" ist damit in ein Zentrum des Interesses, aber auch in einen Sog der Faktizität geraten, den seine Gründungsväter sich nicht ausmalen konnten.

Die administrativen, finanziellen und personellen Probleme dieser Institution, die durch die unerwartete Ausweitung ihrer Aktivitäten entstanden sind und die in unseren Medien immer wieder einmal an die Benutzeroberfläche geholt werden, sollen hier keine Behandlung erfahren. Immerhin hat die Pädagogische Verbindungsarbeit des Goethe-Instituts und der anderen deutschen Mittlerorganisationen heute mehr als 160.000 Deutschlehrer zu betreuen gegenüber etwa $70.000 \mathrm{im} \mathrm{Jahr} \mathrm{1987,} \mathrm{eine} \mathrm{Tat-}$ sache, die größter Anstrengung bedarf, wenn das Goethe-Institut sein Satzungsziel in befriedigender Weise erreichen will.

Es soll auch nichts gesagt werden über das weiterhin latente Mißtrauen gewisser politischer Kreise, denen vor allem das Kulturprogramm, aber auch die Lehrwerke ein Dorn im Auge sind, weil sie den Rat der anfangs zitierten Anzeige nicht beherzigen, die Lautsprecher einfach so aufzudrehen, daß die anderen Nationen Europas von selber merken, daB wir wieder wer sind.

Konzentrieren möchte sich dieser Beitrag vielmehr auf die Auswirkungen des veränderten und sich immer noch verändernden Europas auf die Arbeit des Goethe-Instituts, auf dessen Prioritäten, auf die Maßnahmen und die Probleme, die sich daraus ergeben.

Am Beispiel von zwei Ländern lassen sich die meisten Aspekte stellvertretend darstellen, ein Land im Westen, die Republik Irland, und eines im Osten, nämlich Ungarn. Der Grund für die Auswahl dieser beiden Länder ist ein ganz simpler: Nur über diese beiden Länder liegen aktuelle Berichte vor, die alle Bildungsebenen umfassen, ein statistisch 
zuverlässiges Bild über die Entwicklung des Fremdsprachen-, bzw. Deutschunterrichts geben und relativ aktuell sind. Auch die Aktivitäten des Goethe-Instituts sind in diesen beiden Ländern gerade noch überschaubar, wenn auch nicht unbedingt vergleichbar.

\section{Irland ${ }^{1}$}

In dem kleinen Land ohne nennenswerte Bodenschätze, mit niedrigem Sozialprodukt, konservativen gesellschaftlichen Normen, verletzter nationaler Identität und hoher Arbeitslosigkeit und damit groBem AderlaB durch Auswanderung blieb der Erwerb von Fremdsprachen über Jahrzehnte die Angelegenheit einer kleinen gesellschaftlichen Elite. Analog zu den britischen Schulen spielte einzig Französisch als Fremdsprache eine gewisse Rolle. Deutsch blieb am Rande, bis der Eintritt Großbritanniens in die EG ein Umdenken einlenkte. Anfang der 80er Jahre wurden eine Reform des Fremdsprachenunterrichts an öfentlichen Schulen in Angriff genommen und der Fächerkanon auf Italienisch und Spanisch erweitert. Doch zunächst brachten es die drei zweiten Fremdsprachen zusammen auf nicht mehr als 5-10\%. Noch 1987 lernten zwölfmal mehr Schüler Französisch als Deutsch.

Im Schuljahr 1984/85 gab es nur an 166 von 800 irischen Sekundarschulen Deutschunterricht. Damals schlugen Handel und Industrie Alarm und führten zusammen mit den Universitäten, dem Dublin Institute of Technology und dem National Institute of Higher Education sowie dem Goethe-Institut eine Tagung durch mit dem Titel: „German in Ireland. The Challenge of Business and Technology - The Response of Education", in dessen Folge auf verschiedenen Ebenen Arbeitsgruppen gebildet wurden, die sich Bedarfsermittlung, Lehrpläne und Lehrwerke vornahmen und neue Modelle vor allem im berufsbezogenen Deutschunterricht entwickelten. Von da ab bewegten sich die Vorlieben der Studenten des Tertiärbereichs weg von den klassischen Fächerkombinationen wie Englisch-Deutsch, Französisch-Deutsch, Geschichte-Deutsch hin zu Deutsch plus Wirtschaft, Deutsch plus Europäische Studien, Deutsch plus Statistik. Diese Evolution auf dem Tertiärbereich setzte den Sekundärbereich in Zugzwang, weil die Sprachkenntnisse der Abiturienten meist nicht den Anforderungen der Hochschulen genügten. Im Januar 1988 fand schlieBlich eine Konferenz des National Council for Curriculum and Assessment mit den Erziehungsbehörden, Organen der Industrie und den ausländischen Kulturinstituten statt, das die Fremdsprachenreform

1 Quelle: German in lreland - a complete survey on learning German in lreland. Goethe-Institut Dublin, 1991. 
weiter vorantrieb und folgende Forderungen an das Erziehungswesen erhob:

a) Priorität des Fremdsprachenunterrichts in der Schule

b) Diversifizierung des Fremdsprachenunterrichts

c) solide Finanzierung eines leistungsfähigen Fremdsprachenunterrichts

d) Unterstützung der Reform durch die Behörden, den Handel und die Industrie.

Diese Reform des Fremdsprachenunterrichts hatte zur Folge, daB innerhalb von 8 Jahren der Deutschunterricht an den Sekundarschulen sich verdreifachte. Heute sind es mehr als 400 Schulen, an denen man Deutsch lernen kann. Der Zuwachs vom Schuljahr 1990/91 zum laufenden Schuljahr beträgt ungefähr 20\%. Damit ergeben sich aber weitere Probleme, für deren Lösung das Land die Hilfe des Goethe-Instituts gern in Anspruch nimmt. Es gibt zu wenig Deutschlehrer, viele der vorhandenen Deutschlehrer haben Ausbildungsdefizite, sei es in der Beherrschung der Zielsprache, sei es auf dem berufspraktischen Bereich der Methoden. Für die neuen Fächerkombinationen an den Hochschulen und in der Industrie werden neue Unterrichtsmaterialien benötigt.

Die Bundesrepublik Deutschland unterstützt den Deutschunterricht in Irland durch Entsendung von 5 Deutschlektoren des DAAD an 5 Hochschulen, eines Fachberaters durch die ZfA (Zentralstelle für Auslandsschulen) in das irische Erziehungsministerium, von 14 Assistenten durch den Pädagogischen Austauschdienst und die Gewährung von zahlreichen Stipendien für Schüleraustausch und zur Aus- und Fortbildung von Deutschlehrern. Dazu kommen die Aktivitäten des Goethe-Instituts, das in den vergangenen Jahren an folgenden Projekten mitgewirkt hat:

a) an einer Bedarfsstudie durch Konrad Schröder von der Universität Augsburg;

b) an allen wichtigen Tagungen zur Reform des FSU; insbesondere wurden eine Reihe von Seminaren zur curricularen Planung vom GoetheInstitut initiiert;

c) an vielen Aktivitäten des irischen Deutschlehrerverbands, insbesondere im Zusammenhang mit Lehrerfortbildung;

d) durch die Betreuung einer Arbeitsgruppe zur Erstellung von fachsprachlichen Unterrichtsmaterialien;

e) bei der Erstellung von audiovisuellen Unterrichtsmaterialien für verschiedene Lernergruppen. 
Alle diese Aktivitäten wurden in enger Kooperation mit dem jeweiligen irischen Partner durchgeführt, wobei die Initiativen mal von der einen mal von der anderen Seite ausgegangen sind.

Ungarn ${ }^{2}$

Daß von Ungarn verläBlichere Zahlen vorliegen als von den anderen Ländern Mittel- und Osteuropas, liegt einerseits an der Überschaubarkeit des relativ kleinen Landes, andererseits aber daran, daß die Wende und damit die Reformprozesse dort früher eingesetzt haben als anderswo. Trotzdem ist die Problemlage wesentlich verwickelter, als sie in irgendeinem westeuropäischen Land sein kann.

Zur Vorinformation einige statistische Zahlen:

Mittelschulen:

\section{Englisch \\ Französisch \\ Deutsch \\ Russisch}

Gymnasien:
107799

7864

165923

476286
Russisch
Englisch
Französisch
Deutsch

1990/91 40600 Schüler Englisch in der ersten Klasse

1990/91 40400 Schüler Deutsch
75892

70993

15649

54118

Hochschulen ohne Philologien:

\author{
Englisch \\ Französisch \\ Deutsch \\ Russisch
}

$\begin{array}{lr}\text { Englisch } & 23972 \\ \text { Französisch } & 2494 \\ \text { Deutsch } & 13763 \\ \text { Russisch } & 7071\end{array}$

2 Quelle: Tóth, Pál (1991): Studie zum gegenwärtigen Stand der sprachlichkommunikativen und soziokulturellen Qualifikation von Studenten und Hochschullehrern in Ungarn. Budapest. 
Diese Zahlen erlauben noch kein genaues Bild, was die tatsächliche Entwicklung der einzelnen Sprachen betrifft, da die Statistik noch eine ganze Reihe von Schülergruppen mitführt, die vor der Wende eingeschult worden sind.

Zum Verständnis der Entwicklung lohnt sich ein Blick auf die Zeit vor der Wende zu Beginn der achtziger Jahre, die Pál Tóth als Phase der zentralen Planung und Ausführung wie folgt beschreibt:

„Diese Periode weist gemeinsame Züge in den ehemaligen sozialistischen Ländern auf. Lehrpläne, Lehrbücher und Methoden gab es nach zentralem MaBstab. Das landespädagogische Institut und der Lehrbuchverlag waren Vollzugsorgane des Einheitswillens. Russisch war Pflichtsprache auf allen Ebenen der Ausbildung, in den achtklassigen Grundschulen für lange Zeit die einzige Fremdsprache. Auch heute noch gültig ist das staatlich anerkannte System von Sprachprüfungen mit drei Stufen, das die Anforderungen und Zielsetzungen des FU praktisch in ganz Ungarn bestimmt. Bestimmte Aspekte des FU wie systematische Lehrerfortbildung, zeitgemäße Forschung in Didaktik-Methodik, entsprechende fachliche Foren, Kontakt mit dem westlichen Ausland fehlten."

Die Wende brachte enorme Schwierigkeiten, die sich am bittersten bei den Schülern und Studenten auswirkten. Eine Schülerin der siebten Klasse beschreibt die desolate Situation: „In der 4. Klasse haben wir mit dem Russischen angefangen. Als es abgeschafft wurde, habe ich in der fünften Französisch gelernt. Die Französischlehrerin hat aber am Ende des Jahres gekündigt, und uns wurde angeboten, entweder Deutsch oder Englisch zu lernen. Da es aber in der 6. Klasse keinen Anfängerkurs gab, muBten wir in die Gruppe der Fortgeschrittenen. Es gab einmal in der Woche Nachhilfestunden: wir mußten die Vokabeln im Wörterbuch nachschlagen und die Texte auswendig lernen. Im Sommer habe ich einen Kurs in Englisch besucht, um Schritt halten zu können, wir arbeiteten aber mit "Project English" und das ist sehr schwer. lch lerne auBerdem noch das zweite Jahr Latein. Wir nehmen das Gymnasiallehrbuch."

Durch die Wende wurden die Erziehungsbehörden mit folgenden Tatsachen konfrontiert:

1. Der bisher nach Osten orientiert Fremdsprachenunterricht wandte sich in Richtung Westen.

2. Das gesamte Bildungswesen erfordert eine radikale Umwälzung.

3. Die alten Lehrpläne müssen außer Kraft gesetzt werden.

4. Sämtliche Lehrbücher müssen neu geschrieben werden.

5. Russischlehrer werden überflüssig und müssen umgeschult werden. 
6. Wegen des Wegfalls von Russisch entsteht ein enormer Bedarf an Lehrern für die anderen Fremdsprachen.

Es ist nicht verwunderlich, wenn in einer solchen Situation ein geplantes Vorgehen kaum möglich ist. Pál Tóth beschreibt das Tohuwabohu wie folgt: „Entscheidungen müssen im allgemeinen schnellstens getroffen werden ohne die nötige Vorbereitung. Empirisch fundierte Untersuchungen fehlen vollkommen. Voreiligkeit, manchmal sogar Kopflosigkeit sind zu spüren. Man arbeitet immer unter Zeitdruck, es gibt keine Zeit zu reflektieren, neue Konzepte in fachkundigen Diskussionen auszutragen, Alternativen zu erwägen, auch schon deshalb nicht, weil die nötigen Foren sehr oft fehlen. Ausschlaggebend sind der politische Instinkt oder die „vox populi" wie zum Beispiel im Falle der Abschaffung des Russischunterrichts in den Schulen. Initiativen bleiben oft im Verbalstadium stecken, durch den ständigen Wechsel von Beamten in den verantwortlichen Positionen können Entscheidungen nicht umgesetzt werden. Manchmal wird die unkritische Übernahme fertiger europäischer Modelle gefordert, denn - so wird argumentiert - ist das immer noch besser als das, was wir selber in kurzer Zeit leisten könnten. Man übernimmt das Endprodukt ohne die dazugehörenden „Wurzeln” ohne das "know how”. Es fehlen verantwortungsvolle neue Konzeptionen, mit denen nicht das Ausland kopiert, sondern eigene, der gesellschaftlichen und bildungspolitischen Wirklichkeit in Ungarn entsprechende Maßstäbe gesetzt werden."

Das Goethe-Institut nahm in Ungarn relativ früh seine Tätigkeit auf durch die Gründung eines Kulturinstituts im Jahr 1988 und konnte dort erste Erfahrungen sammeln, die für den späteren Beginn seiner Aktivitäten in den anderen Ländern dieser Region von größtem Nutzen waren. Vor allem waren es die Gründung von Arbeitsgruppen zur Regionalisierung von deutschen Lehrwerken und die Umschulung von Russischlehrern, die an das Goethe-Institut herangetragen wurden. Als besondere Problemlage erwies sich die Tatsache, daB neben den DAADLektoren und den Fachberatern der ZfA zahlreiche Lehrer und Berater im Auftrag der deutschen Bundesländer, der Republik Österreich und sogar aus der Schweiz auftauchten und oft parallel tätig wurden, ohne daß die anderen davon wußten. Die oben beschriebene Konfusion des ungarischen Bildungswesens spiegelte sich in der unkoordinierten Tätigkeit der westlichen Helfer wieder. Ja selbst innerhalb des Goethe-Instituts war davon in den vergangenen zwei Jahren zu spüren. Eine konzeptionell klare Prioritätensetzung war allein deshalb nicht möglich, weil die Partner selber unsteten Witterungsbedingungen ausgesetzt waren und anfangs auch ihre Helfer mit hineinzogen. Mit der Stabilisierung zumindest in Ungarn, aber auch in der CSFR und mit Einschränkung auch 
in Polen beginnt das Goethe-Institut seine neue Rolle zu verstehen, die sich langfristig auch auf die Tätigkeit in Westeuropa auswirkt:

1. Der eigene Unterricht im Ausland dient dem Auf- und Abbau eines Netzwerkes von Institutionen, die einen qualifizierten Deutschunterricht für erwachsene Lerner anbieten. Dazu gehört auch die Entwicklung und Erprobung neuer Lehr- und Selbstlernmaterialien.

2. Die Pädagogische Verbindungsarbeit findet in engem Zusammenspiel mit den Behörden und anderen Partnern im Gastland statt. Sie sucht, eine möglichst breite multiplikatorische Wirkung zu erzielen durch Mitarbeit an Curricula und Lehrwerken für den Unterricht auf allen Ebenen. Dazu gehört auch die Aus- und Fortbildung von Deutschlehrern aller Art und die Einführung von Fremdsprachenunterricht im Grundschulbereich in vielen Ländern Europas.

3. Durch enge Zusammenarbeit mit den Universitäten im Inland und Ausland und durch eigene Publikationen arbeitet das GoetheInstitut an der Standortbestimmung eines modernen Fremdsprachenunterrichts mit. Das bedeutet auch Beobachtung der internationalen Entwicklung und der Zusammenarbeit mit den Mittlerorganisationen anderer europäischer Länder. (Entsendung von Mitarbeitern des GI in das Lingua Büro, Pariser Werkstattgespräche mit dem CREDIF und British Council, binationales Institutsleitertreffen u.a.)

4. Der erhöhte Bedarf an Fremdsprachenunterricht und der damit verbundene Lehrermangel verleiht der Selbstinstruktion vermehrte Bedeutung. Besonders dem Unterricht mit Hilfe der Massenmedien in Radio- und Fernsehsprachkursen, aber auch dem Fernstudium gilt die erhöhte Aufmerksamkeit des Goethe-Instituts. (Radiosprachkurs zusammen mit der Deutschen Welle und dem Deutschlandfunk mit weltweiter Ausstrahlung, Fernsehsprachkurs „Alles Gute” für Erwachsene, in Arbeit Fernsehsprachkurs für Primarschulkinder mit dem WDR, Fernstudienbriefe zusammen mit DIFF und Gesamthochschule Kassel für Germanistik, Landeskunde und MethodikDidaktik)

5. Zur zuverlässigen Evaluierung des Unterrichts gehört ein ausgearbeitetes Prüfungswesen. Zentrale Prüfungen auf allen Niveaus, einschließlich von Prüfungen zur Ermittlung der Vermittlerkompetenz von Deutschlehrern werden weltweit angeboten und erfreuen sich wachsender Nachfrage. Allein in Griechenland wurden 1991 durch die Goethe-Institute 7.519 zentrale Prüfungen abgenommen gegenüber $4389 \mathrm{im} \mathrm{Jahr} \mathrm{1986.} \mathrm{Wo} \mathrm{der} \mathrm{Wunsch} \mathrm{besteht,} \mathrm{wirkt} \mathrm{das}$ Goethe-Institut bei der Entwicklung und Fortschreibung von regionalen und lokalen Prüfungen und Abschlüssen mit. 
Zum Ende dieser Ausführungen sollten einige Problemfelder nicht unerwähnt bleiben:

1.

Das Goethe-Institut verfügt über erhebliche Mittel zur Werbung für den Deutschunterricht. Nicht selten ist damit auch die Frage verbunden, ob nicht wenigstens in einigen Ländern versucht werden sollte, Deutsch mit dem Ziel zu födern, daB Englisch aus seiner Vormachtstellung verdrängt würde. Ganz abgesehen davon, daß Werbung erfahrungsgemäB nur da greift, wo sich ohnehin schon entsprechender Bedarf artikuliert hat, sollte man nicht in den Fehler verfallen, im Sinne der anfangs zitierten Anzeige zu denken. Man sollte auf keinen Fall versuchen, mehr Deutsch durchzusetzen, ohne daß damit eine allgemeine Diversifizierung des Fremdsprachenunterrichts verbunden ist, d.h. mehr Fremdsprachen in der Schule, früherer Beginn mit der ersten Fremdsprache und mehr Unterrichtsstunden überhaupt. Das kann nur erreicht werden, wenn zumindest den Sprachen der jeweiligen Nachbarländer neben den jeweils etablierten Sprachen eine Chance eingeräumt wird. Hier tritt das Goethe-Institut mitunter als Anwalt der "kleineren" Sprachen auf, indem es Tandemkurse durchführt oder unterstützt, wie etwa in Spanien oder auf der griechischen Insel Skopelos, wo alljährlich Griechen und Deutsche gemeinsam jeweils die Sprache des anderen lernen.

2.

Wo mehr Deutsch und andere Fremdsprachen aus überwiegend beruflichen Gründen gelernt werden, gerät der kulturelle Aspekt in den Hintergrund. Die sofortige Anwendbarkeit der gelernten Sprache und deren Umsetzung in wirtschaftlichen Mehrwert läBt aber wenig Platz für das, was beim Erlernen einer Fremdsprache Spaßs macht, nämlich die Beschäftigung mit der Kultur, die hinter dieser Sprache steht. Eine Studie in Großbritannien hat ergeben, daB der überwiegende Teil der Deutschlerner wirtschaftliche Motive verfolgt, während Französischlerner eher das kulturelle und schöngeistige Pläsier im Auge haben. Damit laufen Lehrwerksautoren Gefahr, dem nüchternen Sachtext Vorzug zu geben und die kulturelle Komponente zu vernachlässigen.

3.

Andererseits droht interessanterweise von der Seite der kulturellen Traditionalisten besondere Gefahr. Der Deutschunterricht stagniert dort im öffentlichen Schulwesen, wo eine konservative Germanistik für die Ausbildung von Deutschlehren verantwortlich ist, wie etwa in Frankreich, in Italien und in Belgien. Dort wird geleugnet, daB der moderne Deutschlehrer neben seiner Fachkompetenz auch eine Vermittlungskompetenz benötigt, die es ihm ermöglicht, lebendiger, klarer und motivierender zu 
unterrichten. Deshalb gibt es in diesen Ländern kaum Methodik-Didaktik Veranstaltungen während des Studiums, auch fehlen noch weitgehend berufspraktische Ausbildungsgänge. Diese Gefahr besteht auch da und dort in den osteuropäischen Ländern, wo Traditionalisten ein praxis- und handlungsorientiertes Lehrerstudium zu verhindern versuchen. Nur wenn es dort gelingt, die konservativen Germanisten mit den Reformern, die mehr Methodik-Didaktik und die entsprechenden Bezugswissenschaften in die Studienpläne einbringen möchten, zu versöhnen, wird Deutsch auf lange Sicht die erwartete Position einnehmen können. Deshalb gilt im Moment unser ganzes Bemühen, in Abstimmung mit dem DAAD, der ZfA und den Fachberatern aus Österreich und aus der Schweiz den Reformern in den Staaten Mittel- und Osteuropas den Rücken zu stärken, aber sie gleichzeitig von der Bedeutung der kulturellen Komponente für das gemeinsame europäische Haus und der darin notwendigen Verständigung zu überzeugen.

Der Beirat Deutsch als Fremdsprache am Goethe-Institut unter der Ägide von Harald Weinrich hat den Vermittlern der deutschen Sprache im Ausland 25 Thesen zur Sprach- und Kulturvermittlung ins Stammbuch geschrieben, von denen die folgenden drei hier ihre besondere Relevanz haben:

Nr. 11

Für die Kultur im Sinne der auswärtigen Kulturpolitik ist die Sprachkultur zentral. Unter den Künsten, verstanden als ausgezeichnete Äußerungen der Kultur, hat die Literatur in diesem Rahmen einen besonderen Stellenwert, da sie der Sprachkultur näher steht als andere Künste. Aber auch der Sprachgebrauch außerhalb der Literatur, beispielsweise in den Fachsprachen, steht unter dem Postulat der Sprachkultur.

Nr. 18

Die Begegnung mit der fremden Kultur beginnt in der ersten Stunde des Sprachunterrichts. Von daher ist eine Abtrennung der Spracharbeit von der Kultur nicht angemessen. Vielmehr muß die Verschränkung von Kultur und Sprache Konsequenzen haben für die Entwicklung von Vermittlungsmethoden und Lernkonzepten sowie für Lehrmaterialien.

Nr. 25

Kultur im Sprachbetrieb heißt immer auch: Heiterkeit, Leichtigkeit, Neugierde, Phantasie, Entdeckerfreude. 
Wie es nicht sein sollte, hat Brecht in seinem „Schweyk im 2. Weltkrieg” in eine treffende Anekdote gegossen:

Schweyk: Kennens den: von der Karlsbrücken aus hört ein Tschech ein deitschen Hilfeschrei aus der Moldau. Er hat sich nur iber di Bristung gehengt und hinuntergerufn. "Schrei nicht, hättst schwimmen gelernt statt deitsch!"

(aus: Brecht, Stücke. 5. Band „Schweyk im 2. Weltkrieg”) 